\title{
PERFIL EPIDEMIOLÓGICO DOS PACIENTES POLITRAUMATIZADOS COM PNEUMONIA ASSOCIADA À VENTILAÇÃO MECÂNICA NA UNIDADE DE TERAPIA INTENSIVA DO HOSPITAL UNIVERSITÁRIO EVANGÉLICO DE CURITIBA
}

\author{
EPIDEMIOLOGY OF POLYTRAUMA PATIENTS WITH VENTILATOR- \\ ASSOCIATED PNEUMONIA IN THE INTENSIVE CARE UNIT OF EVANGÉLICO \\ UNIVERSITY HOSPITAL FROM CURITIBA
}

DOI: $10.5380 /$ rmu.v1i1.40680

Matheus Lenci Marques', Vinícius Panichi Musial', Alessandra Amatuzzi Cordeiro2, Marcelo Kuzmicz'

\section{RESUMO}

Resumo: Objetivo: demonstrar o perfil epidemiológico dos pacientes politraumatizados que desenvolveram pneumonia associada à ventilação mecânica na unidade de terapia intensiva do Hospital Universitário Evangélico de Curitiba Materi-al e Método: Foram avaliados os pacientes que desenvolveram pneumonia associada à ventilação mecânica no período de março de 2012 até setembro de 2012, onde foram pesquisados fatores que poderiam influenciar na ocorrência da doença, como sexo, idade, presença de comorbidades, tempo de ventilação mecânica e tempo de internamento. Resultados: Foram avaliados 40 pacientes, com idades entre 18 e 73 anos, sendo 28 (70\%) do sexo masculino e 12 (30\%) do sexo feminino. O tempo de internação dos pacientes em UTI variou de um mínimo de 4 dias para um máximo de 111 dias, com média de 19,1 dias, o tempo de ventilação mecânica variou de 4 a 111 dias, com média de 16 dias. Conclusão: o perfil epidemiológico do paciente politraumatizado que desenvolve pneumonia associada a ventilação mecânica no Hospital Universitário Evangélico de Curitiba é de pacientes do sexo masculino, com idade média de 36 anos, tabagistas, que já fazem uso de ventilação mecânica por pelo menos 16 dias, sendo que a infecção se inicia em média no sexto dia após a intubação do paciente.

Palavras-Chave: Pneumonia associada a ventilação mecânica; Politraumatizado; Unidade de Terapia Intensiva

\section{ABSTRACT}

Abstract: Purpose: Demonstrate the epidemiological profile of polytrauma patients who developed ventilator-associated pneumonia in the intensive care unit of Evangélico University Hospital from Curitiba. Material and Method: Were assessed patients who developed ventilator-associated pneumonia in the period of March 2012 through September 2012. Were surveyed several factors that could influence the occurrence of the ventilator-associated pneumonia, such as gender, age, comorbidities, duration of mechanical ventilation and length of hospitalization. Results: Forty patients aged between 18 and 73 years, with 28 (70\%) males and $12(30 \%)$ female were evaluated in this study. The hospitalization of the patients in the ICU ranged from a minimum of 4 days to a maximum of 111 days with a mean of 19.1 days, the duration of mechanical ventilation ranged from 4 to 111 days with an average of 16 days. Conclusion: The epidemiological profile of polytrauma patients that develops ventilator-associated pneumonia in Evangélico University Hospital from Curitiba demon-strate that this kind of infection predominates in male patients with a mean age of 36 years, smokers, who already make use of mechanical ventilation for at least 16 days and also that infection begins on average in the sixth day following the patient intubation.

Key words: Ventilator-associated pneumonia; Polytrauma; Intensive Care Unit.

1- Faculdade Evangélica do Paraná

2- Pontifícia Universidade Católica do Paraná

Contato do Autor / Mail to:

Matheus Lenci Marques - m.marques133@gmail.com

Rua Padre Anchieta 2770 - Bigorrilho, Curitiba - PR, 80730-000 


\section{INTRODUÇÃO}

Pneumonia é um processo inflamatório que ocorre nos pulmões, podendo ser causado por infecções virais, fungicas, bacterianas e parasitárias ou pela aspiração de conteúdos gástricos ou da orofaringe. Ela pode ser dividida em pneumonia adquirida na comunidade ou pneumonia nosocomial, caracterizada por horas após a internação do paciente ${ }^{1}$. No ano de 2007 esta entidade clínica foi responsável 733.209 internamentos no Brasil, segundo dados do Sistema de Informações Hospitalares do Sistema Único de Saúde, representando a primeira causa de internação por doença, excluindo-se as causas obstétricas. ${ }^{2}$

A pneumonia associada à ventilação mecânica (PAV), que é caracterizada como sendo a infecção pulmonar adquirida 48 horas após o início da ventilação mecânica ${ }^{3}$, é a infecção mais frequentemente adquirida na unidade de terapia intensiva (UTI), com uma incidência variando entre $6 \%$ e $52 \%{ }^{4,5}$. Esta incidência varia muito de um estudo para o outro devido a falta de consenso sobre os critérios diagnóstico para a confirmação da PAV, pelas diferenças entre as UTIs e entre a população avaliada. ${ }^{4,6}$

Em relação às infecções que envolvem outros órgãos, a infecção pulmonar resulta em taxas de mortalidade que variam entre $20 \%$ e $50 \% .^{7}$

O trauma é a principal causa de morte e incapacidade em indivíduos jovens em todo o mundo, sendo seus principais fatores etiológicos os acidentes de trânsito e de trabalho, as tentativas de suicídio e a violência interpessoal ${ }^{8}$. Em 2004, mais de 5 milhões de mortes ocorreram por conta de eventos traumáticos, sendo que destas aproximadamente um quarto foi em consequência de acidentes de trânsito e por ano a OMS acredita que ocorram 1,5 milhões de mortes por violência, incluindo 800 mil suicídios e 500 mil homicídios $^{9}$. Por conta disso, os eventos traumáticos são responsáveis por diversos internamentos hospitalares no Brasil e no mundo, muitas vezes necessitando até mesmo das unidades de terapia intensiva, já que estes pacientes são submetidos a diversas cirurgias e procedimentos invasivos e muitas vezes podem necessitar até mesmo de ventilação mecânica. ${ }^{10}$

Pacientes politraumatizados que apresentam pneumonia são tidos como dilemas diagnósticos, pois existe uma sobreposição de fatores e diversas características compartilhadas com fatores traumáticos como contusão pulmonar, síndrome da angústia respiratória do adulto, lesões agudas ao parênquima pulmonar, entre outras. O trauma associado a pneumonia em pacientes com suporte de ventilação mecânica pode ocorrer como resultado de uma infecção pré-existente, por aspiração ou pela própria ventilação mecânica prolongada. 0 diagnóstico precoce e o tratamento da pneumonia pode melhorar o prognóstico dos pacientes politraumatizados que necessitam de suporte respiratório ${ }^{10}$. Por todos estes fatores, além de ser responsável por aumento de mortalidade, tem sido demonstrado que a PAV prolonga o tempo de internção e a duração da ventilação mecânica, o que leva a um aumento considerável nos custos do tratamento $0^{6,11,12}$. Além disso, o perfil dos pacientes e os critérios para o diagnóstico da PAV variam muito de uma instituição para a outra ${ }^{3}$. Levando em conta tais características desta doença, destacamos a necessidade de medidas de vigilância com coleta de dados locais para um diagnóstico precoce destes pacientes de risco e melhora de seu prognóstico ${ }^{10}$. Em conta disso, este estudo pretende demonstrar o perfil epidemiológico de pacientes politraumatizados que desenvolveram pneumonia associada à ventilação mecânica internados na unidade de terapia intensiva do Hospital Universitário Evangélico de Curitiba.

\section{METODOLOGIA}

Foram incluídos todos os pacientes adultos politraumatizados, com idade superior a 18 anos, internados na Unidade de Terapia Intensiva do Hospital Universitário Evangélico de Curitiba no período de março de 2012 até setembro de 2012, que tiveram o diagnóstico de pneumonia associada a ventilação mecânica. Foi feito um estudo com a análise dos prontuários para a coleta dos seguintes dados destes pacientes: idade, sexo, realização de cirurgia prévia à entrada na unidade de terapia intensiva, tipo de cirurgia realizada, imunossupressão, uso prévio de antibioticoterapia, diabetes melito (DM), etilismo crônico, insuficiência renal crônica (IRC), obesidade (IMC $\geq 30 \%$ ), tabagismo, doença obstrutiva crônica (DPOC), insuficiência cardíaca (ICC), uso de nutrição enteral, coma ( paciente com Glasgow menor que 9 no momento do início da ventilação mecânica), antibioticoterapia profilática após o início da ventilação mecânica, tempo de permanência em ventilação mecânica, dia de início da pneumonia associa-da à ventilação mecânica e tempo de internação na UTI. ${ }^{13}$

Para o diagnóstico de PAV foram levados em conta critérios clínicos e radiológicos. Como critérios clínicos foi verificada a presença de aumento do número de leucócitos totais, aumento e mudança de aspecto de secreção traqueal, piora ventilatória usando principalmente como referência a relação $\mathrm{PaO} 2 / \mathrm{FiO}_{2}$, febre ou hipotermia e ausculta compatível com consolidação, sempre usando como referência o período anterior à suspeita de PAV . Como critérios radiológicos utilizamos o $\mathrm{RX}$ de tórax à beira do leito mostrando novo infiltrado sugestivo de pneumonia, sempre em relação ao período anterior à suspeita. Não há estudos que documentem o papel da tomografia de tórax no diagnóstico da PAV, mas a mesma pode ser 
empregada considerando-se risco/benefício nos casos onde o RX de tórax é duvidoso ${ }^{14,15}$.

Foram considerados pacientes com imunossupressão aqueles que faziam o uso prévio de corticóides ou outras drogas imunossupressoras e aqueles com HIV ou outra doença que leve a imunossupressão.

\section{RESULTADOS}

Durante um período de quatro meses (março a setembro de 2012), analisamos um total de 62 prontuários de pacientes internados na UTI do Hospital Universitário Evangélico de Curitiba. Foram excluídos dos estudos 22 pacientes por não apresentarem os critérios de inclusão. Assim, dos 62 pacientes verificados foram incluídos 40 pacientes no estudo.

Estes pacientes apresentavam idades entre 18 e 73 anos, sendo a média de 36,3 anos e a idade mais prevalente, 29 anos (5 pacientes $-12,5 \%$ ). Em relação ao gênero, 28 (70\%) são do sexo masculino e 12 (30\%) do sexo feminino.

Vinte e quatro pacientes foram admitidos na UTI intubados ou traqueostomizados (60\%), estando todos em ventilação mecânica. Trinta e sete pacientes realizaram cirurgia prévia $(92,5 \%)$, dentre elas: 13 craniectomias descompressivas $(35,14 \%), 7$ drenagens de tórax $(18,92 \%), 6$ fasciotomias $(16,22 \%), 1$ fixador externo de pelve $(2,7 \%)$ e 10 laparotomias exploratórias $(27,03 \%)$.

O tempo de internação dos pacientes em UTI variou de um mínimo de 4 dias para um máximo de 111 dias, com média de 19,1 dias, sendo que em sua maioria os pacientes permaneceram 8 dias internados $(5$ pacientes - 12,5\%). O tempo de ventilação mecânica variou de 4 a 111 dias, com média de 16 dias e uma prevalência de 5 dias ( 6 pacientes - 15\%).

Vinte e quatro pacientes $(60 \%)$ realizaram antibiótico profilaxia após VM. Dos quarenta pacientes avaliados, vinte e seis (65\%) estavam em estado de coma, com uma Escala de Coma de Glasgow menor ou igual a 9 pontos. Dez pacientes (25\%) possuíam DM como um tipo de comorbidade. Três dos pacientes analisados $(7,5 \%)$ apresentavam DPOC. Oito pacientes (20\%) eram etilistas. Outros três pacientes $(7,5 \%)$ apresentavam ICC como uma de suas comorbidades.

De todos os quarenta pacientes analisados, nenhum deles (0\%) era paciente imunossuprimido e nenhum (0\%) estava fazendo uso prévio de antibióticos. Trinta e nove pacientes $(97,5 \%)$ estavam em nutrição enteral. Vinte e quatro (60\%) do total de pacientes avaliados eram tabagistas. Nove pacientes $(22,5 \%)$ eram obesos, apresentando um IMC maior ou igual a 30.

Em 48h de internação em UTI, três pacientes (7,5\%) apresentaram infecção confirmada. Quarenta pacientes (100\%) desenvolveram PAV e o tempo médio para o desenvolvimento da infecção foi de 6,65 dias (com um mínimo de 2 dias e um máximo de 13 dias), sendo que a maioria deles teve início da infecção confirmado 10 dias após o início da VM (20\% dos pacientes).

\section{DISCUSSÃO}

Como se sabe a pneumonia associada a ventilação mecânica é uma das principais causas de morbidade em pacientes politraumatizados. Por isso, é fundamental que se conheça o perfil dos pacientes que desenvolvem este tipo de quadro ${ }^{10}$.

Os resultados deste estudo demonstraram que os pacientes apresentavam uma idade média de 29 anos, dis-cordando da literatura analisada sobre PAV onde os pacien-tes tinham esta média variando de 41 anos até pacientes com mais de 60 anos $^{5,10,11}$.No entanto, como neste caso os pacientes analisados eram também politraumatizados este dado pode ser explicado, já que adultos jovens representam uma grande parcela dos pacientes que sofrem eventos traumáticos ${ }^{9,16,17}$.

Em relação ao sexo, 28 (70\%) dos pacientes avaliados eram do sexo masculino, confirmando os dados da literatura de que tanto a PAV como os politraumatismos acontecem mais neste gênero ${ }^{5,8,10}$. Trinta e sete pacientes $(92,5 \%)$ realizaram cirurgia prévia, sendo que destes $35,14 \%$ realizaram craniectomia descompressiva, sendo estes fatores de riscos já classificados e bem definidos por outros estudos realizados sobre o tema ${ }^{11,15}$.

O tempo médio de ventilação mecânica dos pacientes que desenvolveram PAV segundo a literatura varia, em média, 4 a 15 dias, o que não discorda dos resultados obtidos neste estudo (16 dias). O mesmo vale para o tempo de internamento do paciente na unidade de terapia inten-siva que, segundo a literatura, pode variar de 12 a 20 dias e no presente estudo, a média foi de 19,1 dias $^{5,6,13}$.

A antibióticoterapia profilática nos casos de pacientes em uso de ventilação mecânica ainda é muito divergente. Alguns estudos mostram o uso profilático como um fator de risco5 enquanto outros o demonstram como um fator protetor ${ }^{13}$. Na unidade de terapia intensiva do Hospital Universitário Evangélico de Curitiba é contraindicado o uso de antibióticoterapia profilática, porém neste estudo foram verificados 24 (60\% da amostra analisada) pacientes que fizeram esta profilaxia por conta de outros procedimentos cirúrgicos que antecederam a ventilação mecânica, não sendo observada qualquer diferença entre o dia de início da PAV em relação aos pacientes que não fizeram qualquer tipo de profilaxia.

O dia de início da PAV segundo diversos estudos varia do quarto dia até o décimo terceiro $\operatorname{dia}^{4,7,18}$, o que não divergiu da data de início observada 
no Hospital Universitário Evangélico de Curitiba que foi no décimo dia na maior parte dos casos.

A maior parte dos pacientes avaliados estavam em coma, valor da escala de Glasgow menor do que 9, no momento no início da ventilação mecânica, representando $65 \%$ do total dos pacientes. Além disso, trinta e nove pacientes ( $97,5 \%$ do total) estavam em regime de nutrição enteral, o que favorece a colonização da orofaringe, aumenta o refluxo gastroesofágico e aumenta a chance de broncoaspiração. Outro fato que deve ser destacado é que todos os pacientes estudados estavam em uso de inibidores da bomba de prótons. Como relatado em outros estudos, todas estas condições citadas acima favorecem o aumento da incidência de PAV13.

Nas comorbidades observadas, dez pacientes (25\%) possuíam diabetes melito, três dos pacientes analisados (7,5\%) apresentavam doença pulmonar obstrutiva crônica, três pacientes (7,5\%) apresentavam insuficiência cardíaca crônica e nove pacientes $(22,5 \%)$ eram obesos, apresentando um IMC maior ou igual a 30. Portanto mais da metade dos pacientes estudados apresentava pelo menos uma ou mais comorbidades, sendo todas estas nitidamente fatores de risco para o desenvolvimento, não apenas da pneumonia associada à ventilação mecânica, como também de diversas outras complicações durante o interna-mento $0^{5,9,10}$.

O etilismo e o tabagismo prévio de pacientes que são submetidos a ventilação mecânica também são reconhecidos como fatores predisponentes para o desenvolvimento de infecção pulmonar ${ }^{7}$. Foram observados oito pacientes com histórico de etilismo $(20 \%)$ e vinte e quatro $(60 \%)$ tabagistas, confirmando a grande incidência destes fatores de risco na ocorrência da PAV.

Nenhum dos pacientes estudados apresentou história de imunossupressão, insuficiência renal crônica ou uso prévio de antibióticos, provavelmente não houve este tipo de relato por conta da amostra selecionada para o estudo ser apenas de pacientes politraumatizados, sendo estes mais jovens e de um diferente grupo de pacientes dos demais estudos analisados.

\section{CONCLUSÃO}

Com os resultados obtidos neste trabalho, pode-se concluir que o perfil epidemiológico do paciente politraumatizado que desenvolve pneumonia associada a ventilação mecânica no Hospital Universitário Evangélico de Curitiba predomina com pacientes do sexo masculino, com idade média de 36 anos, tabagistas, que já fazem uso de ventilação mecânica por pelo menos 16 dias, sendo que a infecção se inicia, em média, no sexto dia após a intubação do paciente.
Em sua grande maioria estes pacientes realizaram algum tipo de cirurgia prévia, encontravamse em coma e em uso de nutrição enteral ao início da ventilação mecânica e apresentavam algum tipo de comorbidade como diabetes melito, obesidade (IMC $\geq$ $30 \%)$, doença pulmonar obstrutiva crônica e/ou insuficiência cardíaca.

\section{CONFLITOS DE INTERESSE}

Não houve conflito de interesses.

\section{REFERÊNCIAS BIBLIOGRÁFICAS}

1. Kuyama K, Sun $Y$, Yamamoto $H$. Aspiration pneumonia: With special reference to pathological and epidemiological aspects, a review of the literature. Japanese Association for Dental Science; 2010 Aug 46(2):102-11.

2. Corrêa RDA, Luiz F, Lundgren C, Pereira-silva JL, Luiz R, Cardso AP, et al. Diretrizes da SBPT Diretrizes brasileiras para pneumonia adquirida na. 2009;2009(6):574-601.

3. Mendes P, Rodrigues DA, Neto C, Rodrigo L, Santos DC, Knibel MF. Pneumonia Associada à ventilação mecânica: epidemiologia e impacto na evolução clínica de pacientes em uma unidade de terapia intensiva. 35(11):1084-91.

4. Joseph NM, Sistla S, Dutta TK, Badhe AS, Parija SC. Ventilator-associated pneumonia: a review. European journal of internal medicine [Internet]. European Federation of Internal Medicine; 2010 Oct [cited 2012 Mar 25];21(5):360-8. Available from: http://www.ncbi.nlm.nih.gov/pubmed/20816584

5. Tejerina E, Frutos-Vivar F, Restrepo MI, Anzueto A, Abroug $F$, Palizas $F$, et al. Incidence, risk factors, and outcome of ventilator-associated pneumonia. Journal of critical care [Internet]. 2006 Mar [cited 2012 Jun 14];21(1):56-65. Available http://www.ncbi.nlm.nih.gov/pubmed/16616625

6. Visnegarwala F, lyer NG, Hamill RJ. Ventilator-associated pneumonia. International journal of antimicrobial agents [Internet]. 1998 Aug;10(3):191-205. Available from: http://www.ncbi.nlm.nih.gov/pubmed/9832280

7. Davis $\mathrm{K}$ a. Ventilator-associated pneumonia: a review. Journal of intensive care medicine [Internet]. 2006 [cited 2012 Mar 15];21(4):211-26. Available from: http://www.ncbi.nlm.nih.gov/pubmed/16855056

8. Gomes E, Moreira D, Chaló D, Dias C, Aragão I, Costapereira A. O DOENTE POLITRAUMATIZADO GRAVE Implicações da Ruralidade na Mortalidade, Incapacidade e Qualidade de Vida. 2011;81-90.

9. Herbert HK, Hyder A a, Butchart A, Norton R. Global health: injuries and violence. Infectious disease clinics of North America [Internet]. 2011 Sep [cited 2012 Sep 10];25(3):653-68, x. Available from: http://www.ncbi.nlm.nih.gov/pubmed/21896365

10. Patel CB, Gillespie TL, Goslar PW, Sindhwani M, Peter-sen SR. Trauma-associated pneumonia in adult ventilated patients. American journal of surgery [Internet]. Elsevier 


\section{ARTIGO ORIGINAL}

Inc.; 2011 Jul [cited 2012 May 23];202(1):66-70. Available from: http://www.ncbi.nlm.nih.gov/pubmed/21497790

11. Leong JR, Huang DT. Ventilator-associated pneumonia. The Surgical clinics of North America [Internet]. 2006 Dec [cited 2012 Mar 10];86(6):1409-29

12. 12.Napolitano LM. Hospital-acquired and ventilatorassociated pneumonia: what's new in diagnosis and treatment? The American Journal of Surgery [Internet]. 2003 Nov [cited 2012 Apr 5];186(5):4-14. Available from: http://linkinghub.elsevier.com/retrieve/pii/\$0002961003 $\underline{004847}$

13. 13.Maria C, Maio DD, Matsuo T. Pneumonia Associada à Ventilação Mecânica em Unidade de terapia intensiva Cirúrgica. 2006;(43):38-44.

14. Motta $\mathrm{D}$, Haringer $\mathrm{DC}$. Pneumonia associada à ventilação mecânica . 2009;37-45.

15. Flanagan PG. Diagnosis of ventilator-associated pneumonia. The Journal of hospital infection [Internet]. 1999 Feb;41(2):87-99.

16. Tien H, Chu PTY, Brenneman F. Causes of death following multiple trauma. Current Orthopaedics [Internet]. 2004 Aug [cited 2012 Oct 2];18(4):304-10.

17. Baker CC. Epidemiology of trauma: the civilian perspective. Annals of emergency medicine [Internet]. 1986 Dec;15(12):1389-91. Available from: http://www.ncbi.nlm.nih.gov/pubmed/3777610

18. Martins R, Guimar DEQ, Rocco R. Prevalência e prognóstico dos pacientes com pneumonia associada à ventilação mecânica em um hospital universitário. 32(4):339-46 